\title{
Recent Techniques for the Detection of $\beta$-Thalassemia: A Review
}

\section{Gourav Mishra*, Rupali Saxena, Amit Mishra and Archana Tiwari}

School of Biotechnology, Rajiv Gandhi Proudyogiki Vishwavidyalaya, Airport Bypass Road, Bhopal, Madhya Pradesh, India

\begin{abstract}
$\beta$-thalassemias are very heterogeneous in nature, with respect to the molecular basis of the disease. This type of disorder is caused by extensive deletions occurring within the $\beta$-globin gene locus, present on chromosome number 11. Due to point mutations, stop codons in the $\beta$-globin mRNA is introduced, thus curbing its normal function. This paper reviews conventional and latest $\beta$-thalassemia detection techniques. Amongst the conventional techniques, the DNA-based piezoelectric biosensors were based on the observation of distinguishable frequency shifts, resulting from the hybridisation between an oligonucleotide probe which was immobilized on a gold electrode of a quartz crystal and the complementary strand; present in solution. Q-primer real time PCR (Q-PCR) system utilises the 5'>3' exonuclease activity of a DNA polymerase, which cleaves the nucleotides and results in fluorescence. Amongst the latest detection methods, the capacitive micro-membrane and surface stress based biosensors require flexible structures, microcantilevers or membranes, where one of their surfaces is functionalized with the probe biomolecules. The interaction with the appropriate target molecules induces surface stress variations and finally changes in the deflection of the structure. In bead based biosensors, the natural immobilization of the beads into the polyacrylamide gel pads allows the beads to acquire unique spatial addresses that is recorded via image, and thus helps in avoiding labeling or color encoding steps. These techniques are more efficient and overcome the disadvantages of conventional techniques that were costly, less sensitive and complex too.
\end{abstract}

Keywords: $\beta$-thalassemia; Piezoelectric biosensors; Q-primer real time PCR; Bead based biosensors; Capacitive micro membrane array

\section{Introduction}

Thalassemia is a form of inherited disorders related with nonfunctional hemoglobin protein. Mutation or deletion in $\alpha$ or $\beta$-globin gene that results in shaping of normal and functional hemoglobin protein, results in formation of defective hemoglobin molecule that ultimately cause anemia [1]. Thalassemia can be classified into two types: $\alpha$-thalassemia and $\beta$ - thalassemia.

$\beta$-thalassemias are very heterogeneous in nature, with respect to the molecular basis of the disease. This type of disorder is caused by extensive deletions occurring within the $\beta$-globin gene locus, present on chromosome number 11. Due to point mutations, stop codons in the $\beta$-globin mRNA is introduced; variety of other mutations also affects the splicing sites and sometimes generate new splicing sites [2]. $\beta$-thalassemia is characterized by absent synthesis of $\beta$-globin chains. Individuals heterozygous for $\beta$-thalassemia are considered as carriers and suffer from severities like hypochromia, microcytosis, increased hemoglobin $\mathrm{A} 2$, and an unbalanced $\alpha / \beta$-globin chain synthesis ratio. The concentration of hemoglobin protein varies in mild anemic cases, as compared to normal persons. Homozygotes or compound heterozygotes have intermediate to severe phenotypes. Intermediate condition is referred to as thalassemia intermediate, while severe condition is referred to as thalassemia major [3].

For the treatment of thalassemia, mainly hyper transfusion is prescribed and administration of iron chelating agents can also ensure a normal life. But these treatments must be life-long, and most families in the developing world cannot afford it due to financial reasons. Bone marrow transplantation is also another option, but difficulty lies in finding donor, generally only one-third of patients find an appropriate HLA-matching donor. Approximately, 200 mutations have been detected in patients with $\beta$-thalassemia that interfere with normal $\beta$-globin gene transcription, RNA processing and translation [4].

It is one of the commonest genetic disorders found in humans and poses an increasing public health problem in the tropical countries, where they occur at a high frequency. The detection of bio-molecules, such as DNA or RNA, is a critical process in medical research and diagnostics. The proper detection method for diagnostics purpose often necessitates detecting several targets simultaneously. Therefore, technologies for performing bio-molecular detection must be able to interrogate several targets at one time; currently molecular diagnosis of $\beta$-thalassemia is carried on by different methodologies [5].

\section{Conventional Detection Approaches}

\section{DNA piezoelectric biosensor coupled with polymerase chain reaction}

The DNA-based piezoelectric biosensors are based on the observation of distinguishable frequency shifts, resulting from the hybridization between an oligonucleotide probe which was immobilised on a gold electrode of a quartz crystal and the complementary strand; present in solution [6-9]. Biosensor technology offers the possibility of monitoring hybridization in real time, and with high selectivity.

This type of biosensor was initially used in the Mediterranean area, where the most common $\beta$-thalassemia mutation observed was that of $\mathrm{C} \rightarrow \mathrm{T}$ substitution in the codon 39 of the gene. This biosensor aimed at detecting codon 39 mutation in the $\beta$-globin gene, exploiting the DNA piezoelectric biosensor principle. In the Mediterranean population, this disease is caused mainly by two mutations: the nonsense $\mathrm{C} \rightarrow \mathrm{T}$

*Corresponding author: Gourav Mishra, School of Biotechnology, Rajiv Gandhi Proudyogiki Vishwavidyalaya, Airport Bypass Road, Bhopal, Madhya Pradesh-462033, India, E-mail: mishragourav88@gmail.com

Received August 14, 2012; Accepted October 03, 2012; Published October 08 2012

Citation: Mishra G, Saxena R, Mishra A, Tiwari A (2012) Recent Techniques for the Detection of $\beta$-Thalassemia: A Review. J Biosens Bioelectron 3:123. doi:10.4172/2155-6210.1000123

Copyright: ( 2012 Mishra G, et al. This is an open-access article distributed under the terms of the Creative Commons Attribution License, which permits unrestricted use, distribution, and reproduction in any medium, provided the original author and source are credited. 
substitution at codon 39 (west Mediterranean area) and the $\mathrm{G} \rightarrow \mathrm{A}$ substitution at position 110 of intron 1 (east Mediterranean area) [10].

Minunni et al. [11] discussed about the detection of $\beta$-globin gene mutation based on hybridisation reaction, which was performed using an optimised piezoelectric biosensor. A particular probe was immobilised on the gold surface, using an optimised procedure [12,13]. The immobilized probe recognized a region of the $\beta$-globin gene where a specific mutation occurs (codon 39). The position of the probe was picked out in the region containing codon 39 , so that the mutation was placed in the centre of the probe sequence. The length of the probe was chosen to be of 25 bases [6,14-16].

Minunni et al. [11] investigated about the interactions of the immobilised probe with a complementary, a non-complementary and mismatch synthetic oligonucleotides. The samples were polymerase chain reaction (PCR)-amplified DNA samples that were extracted from human blood of healthy, $\beta$-thalassemia healthy carriers and thalassemic patients.

$10 \mathrm{~Hz}$ AT-cut quartz crystals $(14 \mathrm{~mm})$ were treated with gold that was evaporated $\left(42.6 \mathrm{~mm}^{2}\right.$ area) on both the sides. For the measurements, the quartz crystal was put up inside a methacrylate cell such that only one side of the crystal was in contact with the solution. The frequency variations were continuously recorded using a quartz crystal analyser. The resonance frequency was recorded by a computer connected to the QCA917 interface. The frequency shifts reported in the paper are the difference between two stable frequency values $( \pm 0.5 \mathrm{~Hz})[11]$.

The sequence of the target oligonucleotides ( 25 mer) complementary to the sequence around codon 39 of the $\beta$-globin gene where the mutation occurs, were immobilised on the gold surface of a piezoelectric quartz crystal. The hybridisation between the immobilised probe and the complementary strand in solution was detected, recording the variations of the crystal frequency (Figure 1).

These DNA-based piezoelectric biosensors have several advantages and may be used as an alternative to gel electrophoresis and to traditional methods of specific DNA sequences detection where labelled probes are required. In the conventional approaches, the probes were labelled by radioisotope such as $32^{\mathrm{P}}$ or fluorescent tags, but these biosensors do not rely on using such labels. Moreover, it consumes fewer amounts of expensive reagents and multiple cycles can be performed under the same experimental conditions. This property comes from the fact that these biosensors may be reused by proper washing, by dissociating the hybrids.

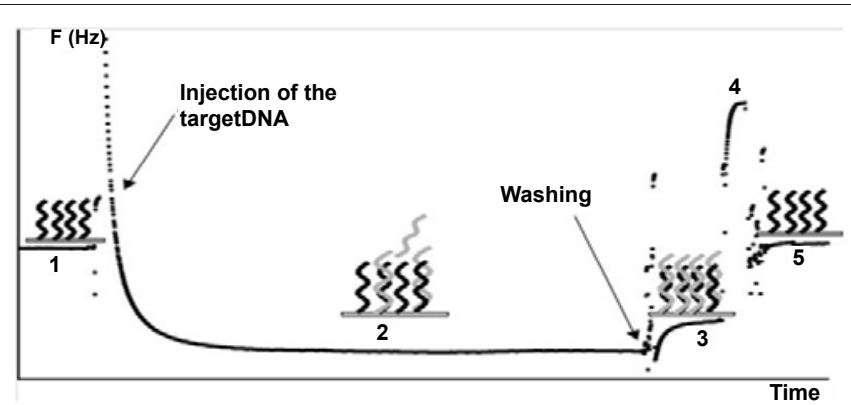

Figure 1: Frequency variations during a hybridisation reaction taking place on the surface of the crystal. The analytical signal is taken as frequency difference between value at point 1 and 3. (1) Free probe; (2) DNA target comes into contact with the free probe; (3) hybridized probe; (4) regenerating agent; and (5) free probe [11].
But it also suffered with the major drawback of being highly expensive, due to the use of quartz crystals that contained gold particles. Even though the biosensor may be reused, hybridised probes, if remain attached, may create false results in further tests [11].

\section{$\beta$-Thalassemia microelectronic chip}

For better detection of highly heterogeneous distribution of molecular defects in the $\beta$-globin gene, Foglieni et al. [17] developed an automated electronic microchip for fast and reliable detection. In this research, the scientist targetted the nine most frequent mutations accounting for $>95 \%$ of the $\beta$-thalassemia alleles in the Mediterranean area. The microelectronic chip system enables deposition and concentration of charged samples to designated test sites on a 100-microelectrode formatted cartridge [18-21]. Arrays consist of biotin-labeled PCR products amplified from patient DNA, and immobilized on test sites through interaction with streptavidin in a permeation layer. Hybridization of specific oligonucleotide probes, complementary to wild-type and mutant sequences, was visualized by fluorogenic indicators.

The $\beta$-globin gene regions (promoter, exons 1 and 2 , introns 1 and 2) containing the mutations of interest, was amplified in four PCR reactions using optimized sets of primers, in order to analyze all nine mutations of interest. The following table shows the location of each amplicon. For each set, one of the primers was 5' biotinylated (Table 1).

For microchip analysis, the scientist used the NMW 1000 NanoChipTM Molecular Biology Workstation and NanoChip cartridges, fabricated by Nanogen Inc. $[17,19]$. Samples were placed on the chip through a loader and electrophoresed by positive bias direct current to selected pads, where they remained embedded through interaction with streptavidin in the permeation layer. The whole process is automated and takes $\sim 3.5 \mathrm{~h} / \mathrm{chip}$. The dye used for labeling wild type and mutant type reporters were 5' Cy3 and 5' Cy5 dye, respectively. But for $-87 \mathrm{C}>\mathrm{G}$, for which 3' 6-carboxytetramethylrhodamine/ Bodipy650/665 labeling of the wild-type and mutant reporters, respectively was used [17].

Complete processing of the chip (100 pads) took approx. 4 hours. Hybridization and fluorescence detection take approx. $20 \mathrm{~min} / \mathrm{chip}$. The study of microelectronic chips provides proper detection of complex heterozygous mutations, causing $\beta$-thalassemia. The scientist also modified their technique by developing several alternative multiplexing features for large-scale screening applications, thus attempting to reduce time and costs. The microelectronic chip system allows the scanning of up to four chips (400 samples) in a single day, thus enhancing the screening of a large number of samples in a highly frequent disease, such as $\beta$-thalassemia. However, use of various dyes i.e. Cy3, Cy5, 6-carboxytetramethylrhodamine/Bodipy650/665 increases the complexity of the process, and also increases the cost. The other drawback is the fact that a convincing amplification of fluorescent signals was obtained when short PCR fragments (up to $200 \mathrm{bp}$ ) encompassing one mutation each, was analyzed individually. Larger DNA fragment resulted in a low signal emission; this happened probably due to the complex secondary structures interfering with

\begin{tabular}{|l|l|l|l|}
\hline Amplicon & Length & Mutation analysis & Mutated alleles detected, \% \\
\hline 1 & 303 & IVS1-110, cd39 & 65.5 \\
\hline 2 & 459 & IVS1-1, IVS1-6, IVS2-1 & 25.7 \\
\hline 3 & 259 & IVS2-745 & 5.2 \\
\hline 4 & 255 & -87, cd6, cd8 & 2.7 \\
\hline
\end{tabular}

Table1: Percentage of mutant $\beta$-thalassemia alleles that can be detected by each amplicon [17]. 
probe hybridization. In fact, as per the facts stated in the paper of Foglieni et al. [17], a single amplicon encompassing all nine mutations did not give sufficiently reliable results.

\section{Allele-specific Q-primer real-time polymerase chain reaction}

Recently, numerous PCR-based homogeneous genotyping assays have been building up for detection purposes. These include DNA binding dye-based methods [22], hybridization probe methods $[23,24]$ and labeled primer methods [25]. But due to some of the shortcomings like amplification of target PCR products as well as nontarget PCR products, primer-dimer products and complexity involved in using more than one dye resulted in adaptation of a new approach, which is based on a novel Q-primer real-time Polymerase Chain Reaction (PCR) system.

In this study, scientist designed allele-specific Q-primers for the detection of three $\beta$-thalassemia mutations [Cd41/42(-TCTT), IVSI nt5 $(\mathrm{G}>\mathrm{C})$, and IVSII nt654 $(\mathrm{C}>\mathrm{T})]$, that have a high carrier frequency in Southeast Asia.

The basic principle of the technique was based on the fact that the 5'>3' exonuclease activity of a DNA polymerase, cleaves the nucleotides that quench BODIPYFL fluorescence at the end of polymerization in a Q-primer real time PCR (Q-PCR) system [26], thereby releasing its fluorescence. As amplification progresses, more fluorescence was emitted. In this experiment, two Q-primers were synthesized which had complementarily resemblance to wild-type and mutant alleles (approx upto 30 nucleotides). One common reverse primer was used in association with the two Q-primers, in separate mutant and wild-type PCRs to probe sample DNA. For heterozygotes, both mutant and wildtype alleles can amplify well, resulting in similar threshold cycle (Ct) values. Samples from homozygous patients generated lower $\mathrm{Ct}$ values from the mutant reaction, than from the wild-type reaction. Thus a genotype can be decided based on difference of Ct values $(\Delta \mathrm{Ct})$ of the two Q-PCRs [27].

For the detection of mutant $\beta$ globin gene in which deletion has occurred, two Q-primers were designed with the 30 differential sequences matching either the wild-type or the deletion mutant. For each sample, two reactions were set up to test the wild-type and mutant alleles, respectively. It was found that differences of $\mathrm{Ct}$ values fall on two distinct groups, with the group with small $\Delta \mathrm{Ct}$ values being heterozygote carriers and the group with large $\Delta \mathrm{Ct}$ values being homozygous non carriers.

This technique requires less time $(2 \mathrm{~h})$ as compared to previous techniques, like Reverse Dot Blot analysis (2 days). A single-vial real-time PCR amplifluor detection system [26] requires use of five oligonucleotides, as compared to two oligonucleotides that are required in Q- primer based PCR. Reaction volume needs only 1.25 pmol of Q-primers per reaction.

As a homogeneous assay, the system can also be potentially adapted in microfluid real-time platforms, and detection of compound $\beta$-thalassemia mutations in close proximity will also need to be demonstrated in the future [27].

\section{Recent Detection Approaches}

\section{Capacitive micro-membrane array}

Previously, analysis of different mutations using microarray serves as a fast and easy genome analysis tool for disease detection [28]. But, this technology is expensive and bulky systems are required in order to measure the label signal for detection [29]. Thus, microarrays could not be miniaturized and cannot be used in point of care diagnostics. Moreover, labelling is a costly and time consuming affair. It may also interfere with the interaction between the probe and target molecules. In order to solve these issues, scientists have come up with new kind of biosensors which can be miniaturized and are label-free systems. Such biosensors are electrical biosensors and surface stress based biosensors.

Surface stress based biosensors are usually bimorph microcantilevers with optical or piezo resistive readout [30], or alternatively capacitive micro-membranes [31,32]. Surface stress based biosensors require flexible structures, microcantilevers or membranes, where one of their surfaces is functionalized with the probe biomolecules. The interaction with the appropriate target molecules induces surface stress variations, and finally changes in the deflection of the structure.

Capacitive detection is desirable as it is highly sensitive and requires low power consumption, but is not feasible in cantilever biosensors, as the electrolyte solution causes faradaic currents between the capacitor plates. Therefore, the scientists used a membrane instead of a cantilever, leading to the capacitor plates being sealed from the electrolyte solution, thereby enhancing reliable capacitive detection. These capacitive membranes exploit the variations of the surface stress induced, when the probe molecules on the functionalized surface interact with their target counterparts. The capacitive sensing elements are parts of a $2 \mathrm{D}$ array $(16 \times 16)$ and are composed of an ultra thin silicon membrane, passivated by a Low Temperature Oxide (LTO) layer that also serves for the functionalization of the biosensors. The operation principle of the sensors relies on the membrane deflection capability, and the translation of the deflection changes into capacitance changes. The capacitor plates are the flexible Si membrane that is highly boron doped in order to become conductive, and the fixed phosphor doped counter electrode [33].

The most frequently encountered (over $90 \%$ of total mutations) molecular abnormalities related to $\beta$-thalassemia oligonucleotides are point mutations (substitutions) and short insertions or deletions, limited to a few nucleotides. Among these the group of researchers selected the CD19 mutation. It is a point mutation (substitution $A \rightarrow G$ ) and represents one of the most important and frequent category of mutations on $\beta$-globin gene.

The biosensor experiment was able to differentiate complementary, as well as single base mismatched $\beta$-thalassemia DNA strands. The capacitive biosensor array is a direct and a fast method in studying the biological interactions. It is also very simple, less costly and is of small size. Thus, it may be suitable for point of care applications.

\section{Spatially addressable bead-based biosensor}

Biosensors comprising biofunctionalized microbeads play an important role in bioanalysis and diagnostic purposes. Owing to the property of high surface to volume ratio, the microbeads can bear a larger number of bioprobes compared to conventional 2D surfaces. Bioassays employing this property of 3D surfaces offer several advantages and provide amended performance. It has now become very fruitful to perform bioassay in microfluidic devices due to its feature of fast reaction kinetics and less reagent consumption. Thus, it is very promising to develop biosensors, integrating both microfluidics and microbeads for multiplexing and fast analysis of biomolecules [34].

Six common Southeast Asian $\beta$-globin gene point mutations, 
representing both transitions $(\mathrm{A} / \mathrm{G}, \mathrm{C} / \mathrm{T})$ and transversions $(\mathrm{G} / \mathrm{T}, \mathrm{G} / \mathrm{C})$ were selected for the study, using these spatially addressable bead based biosensors. The sample was taken as $\beta$-thalassemic patient's genomic DNA. Allele-specific probes targeting various regions of the $\beta$-globin gene were surface-bound to polystyrene microbeads. These beads were then sequentially spotted onto a polymer matrix, at the surface of the biosensor. The polymer matrix causes the first set of spotted beads to be immobilized at unique locations (spatial addresses), which was recorded via an image. The process was then repeated for spotting and distinguishing subsequent bead types, with each bead type carrying probes for targeting either the wildtype or mutant allele of a sample. This procedure allows the different probe-bead types to be identified, without the need for prior encoding of the microbeads. Genomic DNA samples were sequentially amplified by duplex-PCR and asymmetric PCR, followed by hybridization and detection. Hybridization was accomplished within $30 \mathrm{~min}$, and the South East Asian $\beta$-globin genotype can be determined within 3 hours, demonstrating its potential as a simple tool for rapid $\beta$-thalassemia carrier screening [35] (Figure 2).

This figure shows the signal intensity from the wildtype and mutant probes used to target each mutation. All seven different samples were correctly genotyped using the device. For heterozygous mutations, signal intensities from the wildtype probes did not differ significantly from that of the mutant probes. In the absence of a mutation, the wildtype probe intensities were significantly higher than that of the mutant probes. For the homozygous IVSII654 mutation, the mutant probe intensity was significantly higher than the wildtype probe. This similarity or significant difference between wildtype and mutant probe intensities allowed correct identification of the heterozygous mutant and homozygous wildtype (or mutant) samples, respectively.

The device does not require time consuming and labor intensive steps like color encoding, for distinguishing between different bead types [35]. The natural immobilization of the beads into the polyacrylamide gel pads allows the beads to acquire unique spatial addresses that is recorded via image, and thus helps in avoiding labeling or color encoding steps.

Working of these biosensors is simple; it may act as easy-to-use tool for rapid (total time $<3 \mathrm{~h}$ ) detection of mutations and may be used for detection of diseases, other than $\beta$-thalassemia. However, there is need for a prior PCR step to generate the amplified targets for hybridization, which might make it less attractive as compared to one-step PCR-based techniques [35].
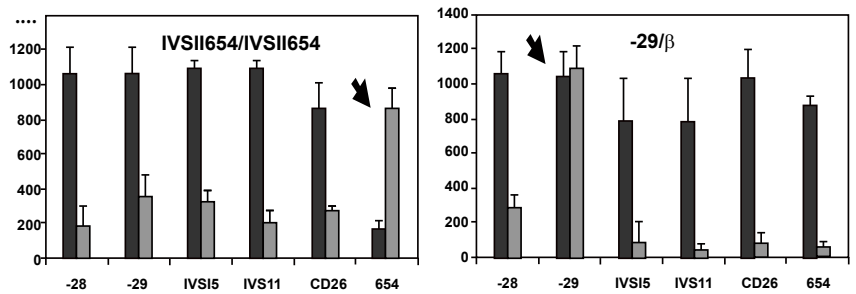

Figure 2: Results of human DNA samples-First graph shows homozygous condition for IVSII654 C $\rightarrow$ T and the second graph shows heterozygous -29 and analyzed using the bead-based biosensor. Signal intensities from the wildtype (I) and mutant (】) probe-bead targeting each mutation [35].

\section{Conclusion}

Hemoglobinopathies (such as $\beta$-thalassemia and sickle cell anemia) are the most common hereditary genetic diseases in the world. They are caused by defects inherent in the gene coding for $\beta$-globin, an essential component of the hemoglobin molecule that carries oxygen in red blood cells from the lungs to body tissue. Different strategies have been reported in this review for detection of $\beta$-thalassemia. Recent techniques like Capacitive Micro-Membrane Array and Bead based Micro Array, combining the concept of biosensors and microfluidics, are proving more beneficial as compared to conventional techniques which were labor intensive, costly and less specific. More and more research needs to be done to design a device that can efficiently detect single base mutation in $\beta$-globin gene. Devices like bead based biosensor and micro-membrane array appear as highly efficient diagnostic devices with tremendous potency. These devices may be used in practical scenario for efficient and quick detection of $\beta$-thalassemia mutations.

\section{Acknowledgments}

I would like to thank Dr. Archana Tiwari for her guidance and valuable inputs.

\section{References}

1. Weatherall D (2006) Thalassemias. Els, John Wiley \& Sons, Ltd.

2. Feriotto G, Breveglieri G, Gardenghi S, Carandina G, Gambari R (2004) Surface plasmon resonance and biosensor technology for real-time molecular diagnosis of beta o 39 thalassemia mutation. Mol Diagn 8: 33-41.

3. Shaji RV, Edison ES, Poonkuzhali B, Srivastava A, Chandy M (2003) Rapid detection of beta-globin gene mutations and polymorphisms by temporal temperature gradient gel electrophoresis. Clin Chem 49: 777-781.

4. Bournazos SN, Tserga A, Patrinos GP, Papadakis MN (2007) A versatile denaturing HPLC approach for human beta-globin gene mutation screening. Am J Hematol 82: 168-170.

5. Johnson KN, Chong S (2011) Multiplexing Capabilities of Biosensors for Clinical Diagnostics. Biosensors for Health, Environment and Biosecurity, Pier Andrea Serra (Ed).

6. Tombelli SM, Mascini M, Sacco C, Turner APF (2000) A DNA piezoelectric biosensor assay coupled with a polymerase chain reaction for bacterial toxicity determination in environmental samples. Analytica Chimica Acta 418: 1-9.

7. Furtado LM, Thompson M (1998) Hybridization of complementary strand and single-base mutated oligonucleotides detected with an on-line acoustic wave sensor. Analyst 123: 1937-1945.

8. Zhou XC, Huang LQ, Li SF (2001) Microgravimetric DNA sensor based on quartz crystal microbalance: comparison of oligonucleotide immobilization methods and the application in genetic diagnosis. Biosens Bioelectron 16: 8595.

9. Deisingh AK, Thompson M (2001) Sequences of E. coli O157:H7 detected by a PCR-acoustic wave sensor combination. Analyst 126: 2153-2158.

10. Rigoli L, Meo A, Miceli MR, Alessio K, Caruso RA, et al. (2001) Molecular analysis of beta-thalassaemia patients in a high incidence area of southern Italy. Clin Lab Haematol 23: 373-378.

11. Minunni M, Tombelli S, Scielzi R, Mannelli I, Mascini M, et al. (2003) Detection of $ß$-thalassemia by a DNA piezoelectric biosensor coupled with polymerase chain reaction. Analytica Chimica Acta 481: 55-64.

12. Tombelli S, Mascini M (2000) Piezoelectric Quartz Crystal Biosensors: Recent Immobilisation Schemes. Anal Lett 33: 2129-2151.

13. Johnsson B, Löfås S, Lindquist G (1991) Immobilization of proteins to a carboxymethyldextran-modified gold surface for biospecific interaction analysis in surface plasmon resonance sensors. Anal Biochem 198: 268-277.

14. Storri S, Santoni T, Minunni M, Mascini M (1998) Surface modifications for the development of piezoimmunosensors. Biosens Bioelectron 13: 347-357.

15. Tombelli S, Mascini M, Braccini L, Anichini M, Turner AP (2000) Coupling of a DNA piezoelectric biosensor and polymerase chain reaction to detect apolipoprotein E polymorphisms. Biosens Bioelectron 15: 363-370. 
Citation: Mishra G, Saxena R, Mishra A, Tiwari A (2012) Recent Techniques for the Detection of $\beta$-Thalassemia: A Review. J Biosens Bioelectron 3:123. doi:10.4172/2155-6210.1000123

Page 5 of 5

16. Minunni M, Tombelli S, Pratesi S, Mascini M, Piatti P, et al. (2001) A Piezoelectric affinity biosensor for genetically modified organisms (GMOs) detection. Anal Lett 34: 825-840.

17. Foglieni B, Cremonesi L, Travi M, Ravani A, Giambona A, et al. (2004) Betathalassemia microelectronic chip: a fast and accurate method for mutation detection. Clin Chem 50: 73-79.

18. Edman CF, Raymond DE, Wu DJ, Tu E, Sosnowski RG, et al. (1997) Electric field directed nucleic acid hybridization on microchips. Nucleic Acids Res 25: 4907-4914.

19. Sosnowski RG, Tu E, Butler WF, O'Connell JP, Heller MJ (1997) Rapid determination of single base mismatch mutations in DNA hybrids by direct electric field control. Proc Natl Acad Sci U S A 94: 1119-1123.

20. Gilles PN, Wu DJ, Foster CB, Dillon PJ, Chanock SJ (1999) Single nucleotide polymorphic discrimination by an electronic dot blot assay on semiconductor microchips. Nat Biotechnol 17: 365-370.

21. Heller MJ, Forster AH, Tu E (2000) Active microeletronic chip devices which utilize controlled electrophoretic fields for multiplex DNA hybridization and other genomic applications. Electrophoresis 21: 157-164.

22. Dall'Ozzo S, Andres C, Bardos P, Watier H, Thibault G (2003) Rapid singlestep FCGR3A genotyping based on SYBR Green I fluorescence in real-time multiplex allele-specific PCR. J Immunol Methods 277: 185-192.

23. Wang MJ, Zhou ZG, Wang L, Li Y, Zhang P, et al. (2009) Genotyping of AKAP10 gene 2073A/G single nucleotide polymorphism by TaqMan probe real-time PCR. Sichuan Da Xue Xue Bao Yi Xue Ban 40: 275-278.

24. Martínez-García A, Sastre I, Tenorio R, Bullido MJ (2004) SNP genotyping with FRET probes. Optimizing the resolution of heterozygotes. Mol Cell Probes 18: 211-214.

25. Myakishev MV, Khripin Y, Hu S, Hamer DH (2001) High-throughput SNP genotyping by allele-specific PCR with universal energy-transfer-labeled primers. Genome Res 11: 163-169.

26. Liu X, Law HY, Tan YM, Hong Y (2010) High-throughput beta-thalassemia carrier screening by allele-specific Q-primer real-time polymerase chain reaction. Anal Biochem 404: 97-99.

27. Liu XK, Hong Y (2007) Q-priming PCR: a quantitative real-time PCR system using a self-quenched BODIPY FL-labeled primer. Anal Biochem 360: 154-156.

28. Shiu SH, Borevitz JO (2008) The next generation of microarray research: applications in evolutionary and ecological genomics. Heredity (Edinb) 100 141-149.

29. Chen X, Sullivan PF (2003) Single nucleotide polymorphism genotyping biochemistry, protocol, cost and throughput. Pharmacogenomics J 3: 77-96.

30. Fritz J (2008) Cantilever biosensors. Analyst 133: 855-863.

31. Cha M, Shin J, Kim JH, Kim I, Choi J, et al. (2008) Biomolecular detection with a thin membrane transducer. Lab Chip 8: 932-937.

32. Tsouti V, Boutopoulos C, Andreakou P, Ioannou M, Zergioti I, et al. (2009) Detection of the biotin-streptavidin interaction by exploiting surface stress changes on ultrathin Si membranes. Microelectronic Engineering 86: 14951498

33. Ng JK, Wang W, Liu WT, Chong SS (2010) Spatially addressable bead-based biosensor for rapid detection of beta-thalassemia mutations. Anal Chim Acta 658: 193-196.

34. Braeckmans K, De Smedt SC, Leblans M, Pauwels R, Demeester J (2002) Encoding microcarriers: present and future technologies. Nat Rev Drug Discov 1: 447-456.

35. Tsouti V, Boutopoulos C, Andreakou P, loannou M, Zergioti I, et al. (2010) Detection of DNA mutations using a capacitive micro-membrane array. Biosens Bioelectron 26: 1588-1592. 